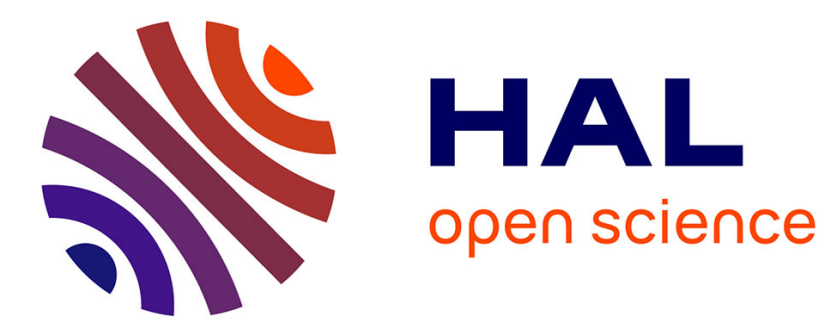

\title{
Correlations of two-dimensional super-paramagnetic colloids in tilted external magnetic fields
}

\author{
Christos N. Likos, Norman Hoffmann, Hartmut Loewen
}

\section{To cite this version:}

Christos N. Likos, Norman Hoffmann, Hartmut Loewen. Correlations of two-dimensional superparamagnetic colloids in tilted external magnetic fields. Molecular Physics, 2007, 105 (13-14), pp.18491860. 10.1080/00268970701441892 . hal-00513110

\author{
HAL Id: hal-00513110 \\ https://hal.science/hal-00513110
}

Submitted on 1 Sep 2010

HAL is a multi-disciplinary open access archive for the deposit and dissemination of scientific research documents, whether they are published or not. The documents may come from teaching and research institutions in France or abroad, or from public or private research centers.
L'archive ouverte pluridisciplinaire HAL, est destinée au dépôt et à la diffusion de documents scientifiques de niveau recherche, publiés ou non, émanant des établissements d'enseignement et de recherche français ou étrangers, des laboratoires publics ou privés. 


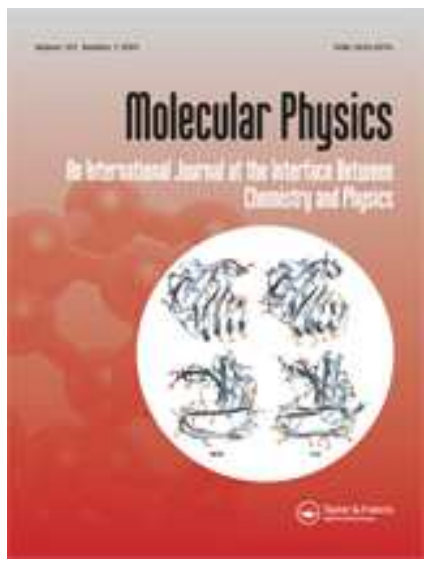

\section{Correlations of two-dimensional super-paramagnetic colloids in tilted external magnetic fields}

\begin{tabular}{|c|c|}
\hline Journal: & Molecular Physics \\
\hline Manuscript ID: & TMPH-2007-0093.R1 \\
\hline Manuscript Type: & Full Paper \\
\hline $\begin{array}{r}\text { Date Submitted by the } \\
\text { Author: }\end{array}$ & 05-May-2007 \\
\hline Complete List of Authors: & $\begin{array}{l}\text { Likos, Christos N.; Heinrich-Heine-University of Duesseldorf, } \\
\text { Institute for Theoretical Physics II } \\
\text { Hoffmann, Norman; Heinrich-Heine Universitaet Duesseldorf } \\
\text { Loewen, Hartmut; Heinrich-Heine-Universitaet Duesseldorf }\end{array}$ \\
\hline Keywords: & $\begin{array}{l}\text { Colloids, Integral equation theories, Liquid structure, Two- } \\
\text { dimensional systems, Anisotropic interactions }\end{array}$ \\
\hline \multicolumn{2}{|c|}{$\begin{array}{l}\text { Note: The following files were submitted by the author for peer review, but cannot be converted } \\
\text { to PDF. You must view these files (e.g. movies) online. }\end{array}$} \\
\hline molphys.tex & \\
\hline
\end{tabular}

\section{scholarONE" Manuscript Central}


Correlations of two-dimensional super-paramagnetic colloids in tilted external magnetic fields

\author{
Norman Hoffmann, Christos N. Likos, ${ }^{*}$ and Hartmut Löwen \\ Institut für Theoretische Physik II: Weiche Materie, \\ Heinrich-Heine-Universität Düsseldorf, \\ Universitätsstraße 1, D-40225 Düsseldorf, Germany
}

(Dated: May 5, 2007)

\begin{abstract}
We outline the formalism of liquid integral equation theory for anisotropic interactions in two dimensions and subsequently apply this theory to one-component super-paramagnetic particles exposed to a tilted magnetic field. Inhomogeneous local ordering of the particles is observed for different in-plane directions. The anisotropy of the interaction as well as of the liquid structure is increased by increasing the tilt angle. Furthermore, the particles favor an alignment in direction of the in-plane component of the magnetic field. For increasing tilt angle, the anisotropy of the structural correlations is qualitatively similar to that of the corresponding solid lattice which is stable at lower temperatures. However, the mean-square displacements behave qualitatively different in the solid and fluid phases as a function of the tilt angle.

PACS numbers: 82.70.Dd, 64.70.Kb, 61.50.Em
\end{abstract}

*Electronic address: likos@thphy.uni-duesseldorf.de 


\section{INTRODUCTION}

While the theory of simple liquids with a radially symmetric pair potential is welldeveloped and established by now [1], anisotropic interactions induce a much richer liquid structure. Both molecular fluids and colloidal suspensions of anisometric particles are typically described by anisotropic pair potentials. The theoretical treatment of anisotropic interactions requires expansions in appropriate spherical harmonics $[2,3]$ and therefore becomes more cumbersome than the radially symmetric case. While the corresponding formalism has been well-developed in three spatial dimensions (see e.g. Refs. [4-6] for some applications) much less is known for two-dimensional fluids with anisotropic pair interactions [7]. The reason for this appears to lie in the fact that strictly two-dimensional systems are not easily realized in nature.

In the past decades, excellent realizations of two-dimensional liquids have been proposed by Maret and coworkers [8-12]. In this set-up, super-paramagnetic colloidal particles are confined by gravity to the two-dimensional air-water interface of a pendant droplet. An external magnetic field which is directed through the droplet surface induces parallel magnetic dipole moments onto the particles, which lead to a dipole-dipole pair interaction among them. The direction of the induced dipole moment coincides with that of the external field and stays fixed, i.e., the dipole moments are not orientable as in complementary studies $[5,13]$. The strength of the induced dipole moment can conveniently be tailored by the magnitude of the external magnetic field. If the magnetic field points perpendicular to the water-air interface, the dipoles repel each other with a pair potential that is proportional to the inverse cube of the interparticle separation. For this system, the fluid structure has been explored in great detail and compared to experiments and simulations [14-16]. However, if the magnetic filed is tilted relative to the surface normal, the interaction becomes anisotropic [17, 18]. If a threshold tilt angle of 54.7 degrees is exceeded the interaction develops an attractive part. Recent theoretical work on tilted fields has been focused on the zero-temperature phase diagram of freezing [19] and on the anisotropic Lindemann parameters in the two-dimensional crystals [20] wherein full agreement was found with the experimental data.

In this paper, we propose a liquid integral equation scheme for anisotropic interactions in two spatial dimensions and apply it in particular to the model potential of magnetic 
colloids in tilted external magnetic fields. We calculate the anisotropic pair correlations for different tilt angles. For a given tilt angle, we compare the local fluid order to that in the solid phase at lower temperature and find that the local fluid ordering reflects that of the crystal. Details like the mean-square displacements, however, are different in the solid and fluid phases. Our liquid integral equation scheme is flexibly applicable also to other twodimensional anisotropic interactions, such as confined colloidal rods or a fluid mono-layer of adsorbed molecules on an attractive surface.

The paper is organized as follows: in section II we define the model. The liquid integral equation scheme is outlined in section III. The results are presented in section IV. Section $\mathrm{V}$ contains a comparison of our results to the solid phase, and finally we conclude in section VI.

\section{THE MODEL}

We consider a two-dimensional system of $N$ colloidal particles in a plane of surface area $A$. With $\rho=N / A$ denoting the system's density we define the characteristic length $a$ as $a \equiv 1 / \sqrt{\rho}$. The particles are exposed to an external magnetic field $\mathbf{B}$ that can be tilted with respect to the two-dimensional plane. This external field induces a magnetic moment $\mathbf{m}_{i}$ onto each particle. The colloids are assumed to be super-paramagnetic, i.e., the magnetic moment of each particle aligns perfectly with the external field:

$$
\mathbf{m}=\chi \mathbf{B}_{0}
$$

with the proportionality constant $\chi$ being the particle's susceptibility. The colloids are of finite extent with a hard core radius $\sigma$. The particles interact with each other via a dipoledipole pair potential, valid for point-like magnetic dipoles,

$$
u_{\mathrm{dd}}\left(\mathbf{r}, \mathbf{m}_{i}, \mathbf{m}_{j}\right)=\frac{\mathbf{m}_{i} \cdot \mathbf{m}_{j}-3\left(\mathbf{m}_{i} \cdot \hat{\mathbf{n}}\right)\left(\mathbf{m}_{j} \cdot \hat{\mathbf{n}}\right)}{r^{3}},
$$

for distances larger than or equal to their hard core radius; $\hat{\mathbf{n}}=\mathbf{r} / r$ is the unit vector connecting the centers of a pair of discs. For distances smaller than the hard core radius the interaction is described by a hard disc repulsion. Using the aforementioned length scale $a$, we introduce the so-called coupling strength, a dimensionless parameter that reads as

$$
\Gamma=\beta \frac{\left(\chi B_{0}\right)^{2}}{a^{3}},
$$




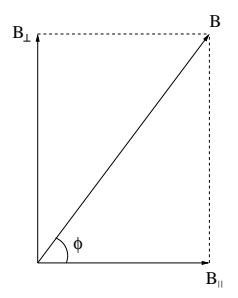

(a)
Molecular Physics

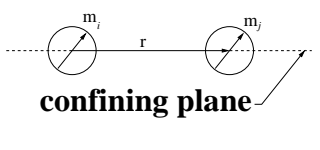

(b)

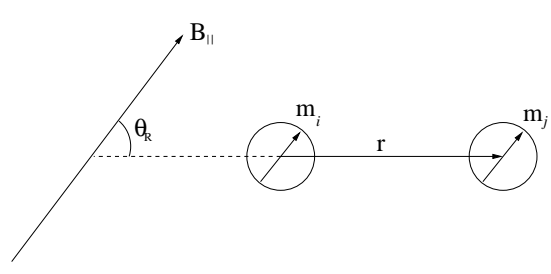

FIG. 1: A schematic view of two super-paramagnetic colloids to a plane exposed to a tilted external magnetic field B: (a) side view; (b) top view.

where $\beta=1 /\left(k_{\mathrm{B}} T\right)$, with $k_{\mathrm{B}}$ being Boltzmann's constant and $T$ being the absolute temperature. Rescaling the particle coordinates with the inter-particle distance, $r_{i} \rightarrow x_{i} \equiv r_{i} / a$, and using eqs. (1) and (3), we can re-write the dipole-dipole interaction potential, eq. (2), as

$$
\beta u_{\mathrm{dd}}(\mathbf{x})=\frac{\Gamma}{x^{3}}\left(1-3 \cos ^{2} \phi \cos ^{2} \theta_{R}\right),
$$

with $\mathbf{x}=\mathbf{x}_{1}-\mathbf{x}_{2}, x=|\mathbf{x}|$, and $\cos \theta_{R}=\mathbf{r} \cdot \mathbf{B}_{\|} /\left(r B_{\|}\right), \mathbf{B}_{\|}$is the in-plane component of the magnetic field; $\phi$ denotes the angle between the magnetic field and its projection to the confining plane. The previously mentioned hard disc diameter $\sigma$ is necessary in order to stabilise the system when the tilt angle increases and attractions in a head-to-tail configuration start appearing. In this study, we will strictly maintain the tilt angle $\phi$ larger than the critical value of 54.7 degrees but keep nevertheless the hard core for completeness. As long as the density $\rho \sigma^{2}$ is small, the particles only see the dipolar potential and the physics is determined entirely by the coupling strength $\Gamma$. In this study, we fixed $\rho \sigma^{2}=0.25$, which is an intermediate value between the dipolar-dominated and packing-dominated regimes. It will be seen that the head-to-tail configurations, for which the repulsion is the weakest, do feel the hard core exclusion.

\section{THEORY}

The radial distribution function $g(\boldsymbol{r})$, or equivalently the total correlation function

$$
h(\boldsymbol{r})=g(\boldsymbol{r})-1
$$


is a quantity of central interest for classical fluids in equilibrium. The radial distribution function measures the extent to which the structure of a fluid deviates from complete randomness and expresses the ordering of the rest of the system around a given particle of the liquid. It plays a key role in liquid theory because some important thermodynamic properties, such as the internal energy of the system, can be written as integrals over $g(\mathbf{r})$ $[1]$.

\section{A. Integral equation theory}

The liquid integral equation theory consists of the Ornstein-Zernike (OZ) relation

$$
h(\mathbf{r})=c(\mathbf{r})+\rho \int \mathrm{d} \mathbf{r}^{\prime} h\left(\mathbf{r}-\mathbf{r}^{\prime}\right) c\left(\mathbf{r}^{\prime}\right)
$$

coupled to a closure relation. We have opted for the hypernetted chain closure (HNC) for reasons to become clear in what follows. The HNC can be written in the form

$$
c(\mathbf{r})=h(\mathbf{r})-\ln g(\mathbf{r})-\beta u(\mathbf{r})
$$

with $c(\mathbf{r})$ denoting the direct correlation function and $\beta u(\mathbf{r})$ the pair interaction potential as explained above. Eqs. (6) and (7) cannot be solved analytically, but a solution can be obtained numerically by an iterative scheme. For convenience we introduce the function

$$
\gamma(\mathbf{r})=h(\mathbf{r})-c(\mathbf{r})
$$

in order to cast the OZ relation in the following form

$$
\gamma(\mathbf{r})=\rho \int \mathrm{d} \mathbf{r}^{\prime}\left[\gamma\left(\mathbf{r}-\mathbf{r}^{\prime}\right)+c\left(\mathbf{r}-\mathbf{r}^{\prime}\right)\right] c\left(\mathbf{r}^{\prime}\right)
$$

For potentials with a hard core part, $h(\mathbf{r})$ and $c(\mathbf{r})$ are separately discontinuous at the hard core radius. The indirect correlation function $\gamma(\mathbf{r})$ is devoid of that discontinuity, and therefore a strictly continuous function. Nevertheless, solving eqs. (7) and (9) is very difficult because each function depends on four variables and the integration in eq. (9) goes over the two position and angular coordinates. In order to simplify and solve the equations, we expand the correlation function into a basis set of orthogonal functions. This expansion leads to an easily tractable form of the OZ equation. Then, it is possible to expand the HNC closure analytically to all orders in the same basis set, hence allowing a solution of eqs. (7) and (9). 


\section{B. Expansion}

Unlike systems with isotropic interactions, in our case the correlation functions depend on both $r \equiv|\mathbf{r}|$ and the angle between $\mathbf{r}$ and the projection of the dipole moment on the $x$-axis, which lies parallel to $\mathbf{B}_{\|}$. Accordingly, an expansion of $h(\mathbf{r})$ and $c(\mathbf{r})$ on orthogonal functions is required. The formalism for three spatial dimensions was developed by Caillol, Weis and Patey [21] for fixed dipoles (as in our case) and involves the spherical harmonics $Y_{l m}(\Omega)$. In two spatial dimensions, Caillol, Levesque and Weis [22] have introduced the expansion which we also utilise in what follows but they focused there on a system with orientable dipoles, rather than fixed ones. Moreover, in Ref. [22] emphasis was put on a restricted set of expansion coefficients of the correlation functions, related to the inetrnal energy of the system. Finally, we combine the expansion with a technique for rapid evaluation of the $m$-th order Hankel trasfromations, which naturally arise in the formalism.

Since we are working in a two-dimensional system, the complex exponential function is a natural choice for an orthogonal basis set to expand the correlation functions $c(\mathbf{r})$ and $h(\mathbf{r})$. Exemplary, we just show the direct correlation function as a representative for all correlation functions

$$
c(\mathbf{r})=\sum_{m} c_{m}(r) \exp \left(-\mathrm{i} m \theta_{R}\right)
$$

with $\theta_{R}$ denoting the angle between $\mathbf{r}$ and the $x$-axis, and identifying $\mathbf{r} \equiv\left(r, \theta_{R}\right)$. Due to symmetry, this expansion can be rewritten as

$$
c(\mathbf{r})=c_{0}(r)+\sum_{|m| \neq 0} c_{|m|}(r) \cos \left(m \theta_{R}\right)
$$

with

$$
c_{|m|}(r)= \begin{cases}\frac{1}{\pi} \int_{0}^{\pi} \mathrm{d} \theta_{R} c(\mathbf{r}) & \text { for } m=0 \\ \frac{2}{\pi} \int_{0}^{\pi} \mathrm{d} \theta_{R} c(\mathbf{r}) \cos \left(m \theta_{R}\right) & \text { for } m \neq 0\end{cases}
$$

where $m$ has to be an even number.

The interaction potential can be expanded in the same basis set as the correlation functions and is fully determined by the first two coefficients with the remaining terms vanishing:

$$
\begin{aligned}
& \beta u_{0}(r)=\beta \frac{\left(\chi B_{0}\right)^{2}}{r^{3}}\left(1-\frac{3}{2} \cos ^{2} \phi\right), \\
& \beta u_{2}(r)=-\beta \frac{3}{2} \frac{\left(\chi B_{0}\right)^{2}}{r^{3}} \cos ^{2} \phi .
\end{aligned}
$$


Solving eqs. (7) and (9) simultaneously requires the expansion of the HNC closure in the same set of basis functions as all the other correlation functions. At first sight, it seems that the logarithmic term in eq. (7) causes trouble, but this problem has been overcome by Fries and Patey [23] as follows: partial differentiation of eq. (7) with respect to $r$ yields

$$
\frac{\partial c\left(r, \theta_{R}\right)}{\partial r}=-h\left(r, \theta_{R}\right) \frac{\partial W\left(r, \theta_{R}\right)}{\partial r}-\beta \frac{\partial u\left(r, \theta_{R}\right)}{\partial r},
$$

introducing $W\left(r, \theta_{R}\right) \equiv-\gamma\left(r, \theta_{R}\right)+\beta u\left(r, \theta_{R}\right)$ as the dimensionless angle-dependent potential of mean force. Now, since $c\left(r, \theta_{R}\right) \rightarrow-\beta u\left(r, \theta_{R}\right)$ as $r \rightarrow \infty$, it is clear that

$$
\int_{r}^{\infty} \mathrm{d} r^{\prime} \frac{\partial c\left(r^{\prime}, \theta_{R}\right)}{\partial r^{\prime}}=\left[c\left(r=\infty, \theta_{R}\right)-c\left(r, \theta_{R}\right)\right]=-c\left(r, \theta_{R}\right) .
$$

Combining eqs. (14) and (15), the HNC closure reads as

$$
c\left(r, \theta_{R}\right)=\int_{r}^{\infty} \mathrm{d} r^{\prime} h\left(r^{\prime}, \theta_{R}\right) \frac{\partial W\left(r^{\prime}, \theta_{R}\right)}{\partial r^{\prime}}-\beta u\left(r, \theta_{R}\right) .
$$

The advantage of writing the HNC closure like this amounts to the analytical expansion of the binary product $-h\left(r, \theta_{R}\right)\left[\partial W\left(r, \theta_{R}\right) / \partial r\right]$ in the common basis set. After some algebra, we obtain

$$
c\left(r, \theta_{R}\right)=\sum_{m} \sum_{n=0}^{m} I_{n, m-n}(r) \exp \left(-\mathrm{i} m \theta_{R}\right)-\beta \sum_{m} u_{m}(r) \exp \left(-\mathrm{i} m \theta_{R}\right),
$$

with

$$
I_{m, n}(r)=\int_{r}^{\infty} \mathrm{d} r^{\prime} h_{m}\left(r^{\prime}, \theta_{R}\right) \frac{\partial W_{n}\left(r^{\prime}, \theta_{R}\right)}{\partial r^{\prime}} .
$$

For distances smaller than the hard core radius $\sigma$ the radial distribution function is zero, hence the direct correlation function can be calculated by means of eq. (8) as

$$
c\left(r, \theta_{R}\right)=-1-\gamma\left(r, \theta_{R}\right) \text { for } r<\sigma
$$

\section{Fourier transform}

In solving the two coupled integral equations, the $\mathrm{OZ}$ and the $\mathrm{HNC}$ closure invoke an iterative procedure that requires the repeated calculation of convolution integrals. These are evaluated most efficiently and rapidly in reciprocal space. Therefore we first transform the OZ relation to reciprocal space in order to render the convolution integral into a simple product. Then, we introduce the expansion of correlation functions in reciprocal space into the OZ relation. 
We define the Fourier transform for an arbitrary, physically smooth function $f(\mathbf{r})$ in $2 \mathrm{~d}$ as follows

$$
\tilde{f}(\mathbf{k})=\int \mathrm{d}^{2} r f(\mathbf{r}) \exp (\mathrm{ik} \cdot \mathbf{r})
$$

and its inverse as

$$
f(\mathbf{r})=\frac{1}{(2 \pi)^{2}} \int \mathrm{d}^{2} k \tilde{f}(\mathbf{k}) \exp (-\mathrm{ik} \cdot \mathbf{r})
$$

The OZ relation (9) can be written in Fourier space as

$$
\tilde{\gamma}(\mathbf{k})=\rho \tilde{c}(\mathbf{k})[\tilde{\gamma}(\mathbf{k})+\tilde{c}(\mathbf{k})]
$$

The expansion in orthogonal functions can be written in Fourier space as

$$
\tilde{c}(\mathbf{k})=\sum_{m} \tilde{c}_{m}(k) \exp \left(-\mathrm{i} m \theta_{K}\right)
$$

with

$$
\tilde{c}_{m}(k)=2 \pi \mathrm{i}^{m} \int_{0}^{\infty} \mathrm{d} r r c_{m}(r) J_{m}(k r) .
$$

Here, $\theta_{K}$ in Fourier space is the equivalent to $\theta_{R}$ in real space. We point out explicitly that the angle $\theta_{R}$ in the real space expansion and the angle $\theta_{K}$ in the reciprocal space expansion are different. In each system, it is the in-plane angle between the in-plane magnetic field and the $r$ - and $k$-vector, respectively. Moreover $J_{m}(r)$ is the $m$-th order Bessel function of the first kind. Conversely, the coefficients of the expansion in reciprocal space are obtained by a simple Hankel transform of the coefficient in real space. Thus, it holds

$$
c_{m}(r)=(2 \pi)^{-1} \mathrm{i}^{m} \int_{0}^{\infty} \mathrm{d} k k \tilde{c}_{m}(k) J_{m}(k r) .
$$

In the appendix, we show a strict derivation of the interconnection between the coefficients in real and Fourier space.

The problem of calculating the Hankel transform of $m$-th order amounts to calculating integrals of the type

$$
\tilde{f}_{m}(k)=\int_{0}^{\infty} \mathrm{d} r \operatorname{rf}(r) J_{m}(k r) .
$$

Such integrals can be reduced to those appearing in the 0-th order Hankel transform,

$$
\tilde{f}_{0}(k)=\int_{0}^{\infty} \mathrm{d} r r f(r) J_{0}(k r) .
$$


Lado developed a numerically accurate algorithm for this procedure [24] and for the calculation of the 0-th order Hankel transform in an efficient way [25]. For the reduction, we need two recurrence relations for the Bessel functions [26],

$$
\begin{aligned}
\frac{\mathrm{d}}{\mathrm{d} x}\left[\frac{J_{m-1}(x)}{x^{m-1}}\right] & =-\frac{J_{m}(x)}{x^{m-1}}, \\
\frac{\mathrm{d}}{\mathrm{d} x}\left[x^{m} J_{m-1}(x)\right] & =x^{m} J_{m-2}(x) .
\end{aligned}
$$

Introducing the first recurrence relation, eq. (28), into eq. (26) and an integration by parts leads to the following expression with $f(r)$ vanishing sufficiently rapidly at infinity:

$$
\tilde{f}_{m}(k)=\frac{1}{k} \int_{0}^{\infty} \mathrm{d} r \frac{\mathrm{d}}{\mathrm{d} r}\left[r^{m} f(r)\right] \frac{J_{m-1}(k r)}{r^{m-1}} .
$$

We set $f(r) \equiv f^{(m)}(r)$ and define a new function $f^{(m-2)}(r)$ such that

$$
\frac{\mathrm{d}}{\mathrm{d} r}\left[r^{m} f^{(m)}(r)\right]=r^{2 m-2} \frac{\mathrm{d}}{\mathrm{d} r}\left[\frac{f^{(m-2)}(r)}{r^{m-2}}\right]
$$

Introducing eq. (31) into eq. (30) leads to

$$
\begin{aligned}
\tilde{f}_{m}(k) & =\frac{1}{k} \int_{0}^{\infty} \mathrm{d} r r^{2 m-2} \frac{\mathrm{d}}{\mathrm{d} r}\left[\frac{f^{(m-2)}(r)}{r^{m-2}}\right] \frac{J_{m-1}(k r)}{r^{m-1}} \\
& =\int_{0}^{\infty} \mathrm{d} r \frac{1}{r} \frac{\mathrm{d}}{\mathrm{d} r}\left[\frac{f^{(m-2)}(r)}{r^{m-2}}\right] \int_{0}^{r} \mathrm{~d} x x^{m} J_{m-2}(k x) \\
& =-\int_{0}^{\infty} \mathrm{d} r r f^{(m-2)}(r) J_{m-2}(k r),
\end{aligned}
$$

where in the first step we used the second recurrence relation, eq. (28), and in the next step we integrated by parts. Eq. (32) allows us, therefore, to express the original, $m$-th order Hankel transformation as a $m-2$-order one but with a new function $f^{(m-2)}(r)$ instead of the original, $f(r) \equiv f^{(m)}(r)$.

To complete the specification, we have to relate the function $f^{(m-2)}(r)$ to its counterpart $f^{(m)}(r)$. Thereafter, we integrate eq. (31)

$$
\int_{r}^{\infty} \mathrm{d} r^{\prime} \frac{\mathrm{d}}{\mathrm{d} r^{\prime}}\left[\left(r^{\prime}\right)^{m} f^{(m)}\left(r^{\prime}\right)\right]=\int_{r}^{\infty} \mathrm{d} r^{\prime}\left(r^{\prime}\right)^{2 m-2} \frac{\mathrm{d}}{\mathrm{d} r^{\prime}}\left[\frac{f^{(m-2)}\left(r^{\prime}\right)}{\left(r^{\prime}\right)^{m-2}}\right] .
$$

Performing the integration yields

$$
f^{(m-2)}(r)=f^{(m)}-2(m-1) r^{m-2} \int_{r}^{\infty} \mathrm{d} r^{\prime} \frac{f^{(m)}\left(r^{\prime}\right)}{\left(r^{\prime}\right)^{m-1}} .
$$


We apply repetitively this 'step-down' operation until we reach

$$
f^{(0)}(r)=f^{(2)}-2 \int_{r}^{\infty} \mathrm{d} r^{\prime} \frac{1}{r^{\prime}} f^{(2)}\left(r^{\prime}\right) .
$$

Then, we also repeatedly apply the reduction of order of the Hankel transformation, eq. (32), until we reach the level of transforming $f^{(0)}(r)$ by a 0th-order Hankel transform [25].

Similarly, it can be shown that for the inverse $m$-th order Hankel transform the following scheme holds:

(1) First, we transform the original function $\tilde{f}(k) \equiv \tilde{f}^{(0)}(k)$ by a simple 0 -th order Hankel transform in order to obtain $f^{(0)}(r)$.

(2) Then, we apply the following expression repetitively until the desired order of $m$ is reached

$$
f^{(m)}(r)=f^{(m-2)}(r)-\frac{2(m-1)}{r^{m}} \int_{0}^{r} \mathrm{~d} x x^{m-1} f^{(m-2)}(x) .
$$

The latter integral as well as the integral in eq. (34) can be calculated using a simple trapezoidal rule.

Because the direct correlation function $c(r)$ behaves asymptotically as the potential,

$$
c(r) \sim-\beta u(r) \sim \frac{1}{r^{3}}, \quad(r \rightarrow \infty)
$$

the long-range nature of the correlation function may adversely affect the Fourier transform due to a necessary truncation of the former one at a finite cut-off value. We can bypass that problem by subtracting a function with equal long-range behavior and an analytically known Hankel transform. The Fourier transform of that difference does not entail any difficulties and the Hankel transform of the subtracted function is added back in reciprocal space.

For the coefficient $c_{0}(r)$, we subtract the function

$$
\begin{aligned}
\beta u_{\mathrm{LR}, 0}(r ; \alpha) & =\frac{\Gamma}{r^{3}}\left[1-\exp (-\alpha r)\left(1+\alpha r+\frac{1}{2} \alpha^{2} r^{2}\right)\right] \\
& =\frac{1}{2} \Gamma \alpha^{3} \int_{0}^{1} \mathrm{~d} x x^{2} \exp (-\alpha r x)
\end{aligned}
$$

whose Hankel transform is analytically known and reads as

$$
\beta \tilde{u}_{\mathrm{LR}, 0}(k ; \alpha)=\pi \Gamma\left[\frac{2 k^{2}+\alpha^{2}}{\sqrt{k^{2}+\alpha^{2}}}-2 k\right] .
$$


For the coefficient $c_{2}(r)$, the following function proves to be apt:

$$
\begin{aligned}
\beta u_{\mathrm{LR}, 2}(r ; \alpha) & =\frac{\Gamma}{r^{3}}\left[1-\exp (-\alpha r)\left(1+\alpha r+\frac{1}{2} \alpha^{2} r^{2}+\frac{1}{6} \alpha^{3} r^{3}\right)\right] \\
& =\frac{1}{6} \Gamma \alpha^{4} r \int_{0}^{1} \mathrm{~d} x x^{3} \exp (-\alpha r x),
\end{aligned}
$$

whose Hankel transform reads as

$$
\beta \tilde{u}_{\mathrm{LR}, 2}(k ; \alpha)=-\frac{\pi}{3} \Gamma k^{2}\left(\frac{2}{k}-\frac{3 \alpha^{2}+2 k^{2}}{\left(\alpha^{2}+k^{2}\right)^{3 / 2}}\right)
$$

In both cases, $\alpha$ is an arbitrary parameter, which we chose as $\alpha=1.25$.

It is worth noting to treat the discontinuity in $c(r)$ carefully by doing the Fourier transform. We apply the Dirichlet condition at this position to guarantee correct results. This condition states that for a function with a discontinuity at $r_{0}$ the Fourier transform at this position is just the Fourier transform of the mean value of the lower and upper value.

\section{Ornstein-Zernike relation}

Our initial goal was to cast the Ornstein-Zernike relation in a numerically tractable form. First, we broke up its integral in a simple product by passing over to Fourier space. Then, we outlined the series expansion in Fourier space. The final step comprises introducing the series expansion in reciprocal space, eq. (23), into the Fourier transformed Ornstein-Zernike relation, eq. (22), yielding the following result:

$$
\tilde{\gamma}_{p}(k)=\rho \sum_{m} \tilde{c}_{m}(k) \tilde{\gamma}_{(p-m)}+\tilde{c}_{m}(k) \tilde{c}_{(p-m)}
$$

Restricting to a limited number $M$ of coefficients in the series expansion, the OrnsteinZernike relation provides a $M \times M$ linear algebraic system which can be solved by standard numerical algorithms.

\section{E. Algorithm}

The elements developed above are necessary ingredients in analyzing the two coupled integral equations (6) and (7). The whole procedure to solve these equations is best presented in summary form. One iteration for calculating the $c_{m}(r)$ consists of the following steps: 
1. Subtract from the coefficients $c_{0}(r)$ and $c_{2}(r)$ the functions

$$
\beta u_{\mathrm{LR}, 0}(r ; \alpha)=\frac{\Gamma}{r^{3}}\left[1-\exp (-\alpha r)\left(1+\alpha r+\frac{1}{2} \alpha^{2} r^{2}\right)\right]
$$

and

$$
\beta u_{\mathrm{LR}, 2}(r ; \alpha)=\frac{\Gamma}{r^{3}}\left[1-\exp (-\alpha r)\left(1+\alpha r+\frac{1}{2} \alpha^{2} r^{2}+\frac{1}{6} \alpha^{3} r^{3}\right)\right],
$$

respectively.

2. Successively lower the coefficients $c_{m}(r)$ to obtain

$$
c_{m}^{(l-2)}(r)=c_{m}^{(l)}-2(l-1) r^{l-2} \int_{r}^{\infty} \mathrm{d} r^{\prime} \frac{1}{r^{\prime l-1}} c_{m}^{(l)}\left(r^{\prime}\right)
$$

commencing with $c_{m}^{(m)}(r) \equiv c_{m}(r)$ and ending up with $c_{m}^{(0)}(r)$.

3. Convert $c_{m}^{(0)}(r)$ by a 0 -th order Hankel transform following the procedure outlined in Ref. [25], to obtain $\tilde{c}_{m}(k)$. Be aware of the discontinuity at the hard core radius and apply the Dirichlet condition described at the end of section III C.

4. Add the analytically calculated Hankel transforms

$$
\beta \tilde{u}_{\mathrm{LR}, 0}(k ; \alpha)=\pi \Gamma\left[\frac{2 k^{2}+\alpha^{2}}{\sqrt{k^{2}+\alpha^{2}}}-2 k\right]
$$

and

$$
\beta \tilde{u}_{\mathrm{LR}, 2}(k ; \alpha)=-\frac{\pi}{3} \Gamma k^{2}\left(\frac{2}{k}-\frac{3 \alpha^{2}+2 k^{2}}{\left(\alpha^{2}+k^{2}\right)^{3 / 2}}\right)
$$

back to the coefficients $\tilde{c}_{0}(k)$ and $\tilde{c}_{2}(k)$.

5. Introduce $\tilde{c}_{m}(k)$ into the Ornstein-Zernike relation

$$
\tilde{\gamma}_{p}(k)=\rho \sum_{m} \tilde{c}_{m}(k) \tilde{\gamma}_{(p-m)}+\tilde{c}_{m}(k) \tilde{c}_{(p-m)}
$$

to obtain $\tilde{\gamma}_{m}(k) \equiv \tilde{\gamma}_{m}^{(0)}(k)$

6. Apply to these the inverse 0-th order Hankel transform to obtain the function $\gamma_{m}^{(0)}(r)$.

7. Raise this function repeatedly according to:

$$
\gamma_{m}^{(l)}(r)=\gamma_{m}^{(l-2)}(r)-\frac{2(l-1)}{r^{l}} \int_{0}^{r} \mathrm{~d} x x^{l-1} \gamma_{m}^{(l-2)}(x),
$$

until the desired function $\gamma_{m}(r) \equiv \gamma_{m}^{(m)}(r)$ is reached. 
for distances larger than or equal to the hard core diameter and

$$
c\left(r, \theta_{R}\right)=-1-\gamma\left(r, \theta_{R}\right)
$$

for distances smaller than that diameter to calculate the new function $c\left(r, \theta_{R}\right) \equiv c(\mathbf{r})$ for the iteration. For the derivative in eq. (50), we applied a 7-point derivative scheme for unevenly spaced nodes [27].

This iterative scheme is repeated until convergence for $c_{m}(r)$ is achieved. To guarantee convergence, we mix the new $c_{m}(r)$ with the result of the former iterative step

$$
c_{m}^{\text {new }}(r)=\zeta c_{m}^{\text {new }}(r)+(1-\zeta) c_{m}^{\text {old }}(r)
$$

The so-called mixing parameter $0<\zeta<1$ is chosen empirically to ensure convergence. The whole iterative scheme is best started with the Mayer function $f(r)$

$$
f(\mathbf{r})=\exp [-\beta u(\mathbf{r})]-1
$$

which is exact in the zero density limit. The above scheme can be started with a small density and is proceeded through the outlined steps until convergence is reached, and then we slightly increase the density, repeat the iterative scheme again and then further increase the density until the desired density is obtained.

\section{RESULTS}

We have numerically solved the two coupled integral equations (eqs. (6) and (7)), according to the theory outlined in the previous section. Here, we present representative results for the correlation functions and their dependence on coupling, tilt angle and angular orientation in the plane.

First, we varied the interaction strength $\Gamma$ for various parameter combinations of the tilt angle $\phi$ and the in-plane angle $\theta_{\mathrm{R}}$. The results are shown in Fig. 2. It can be seen that the peak heights increase with increasing interaction strength while the peak positions remain 

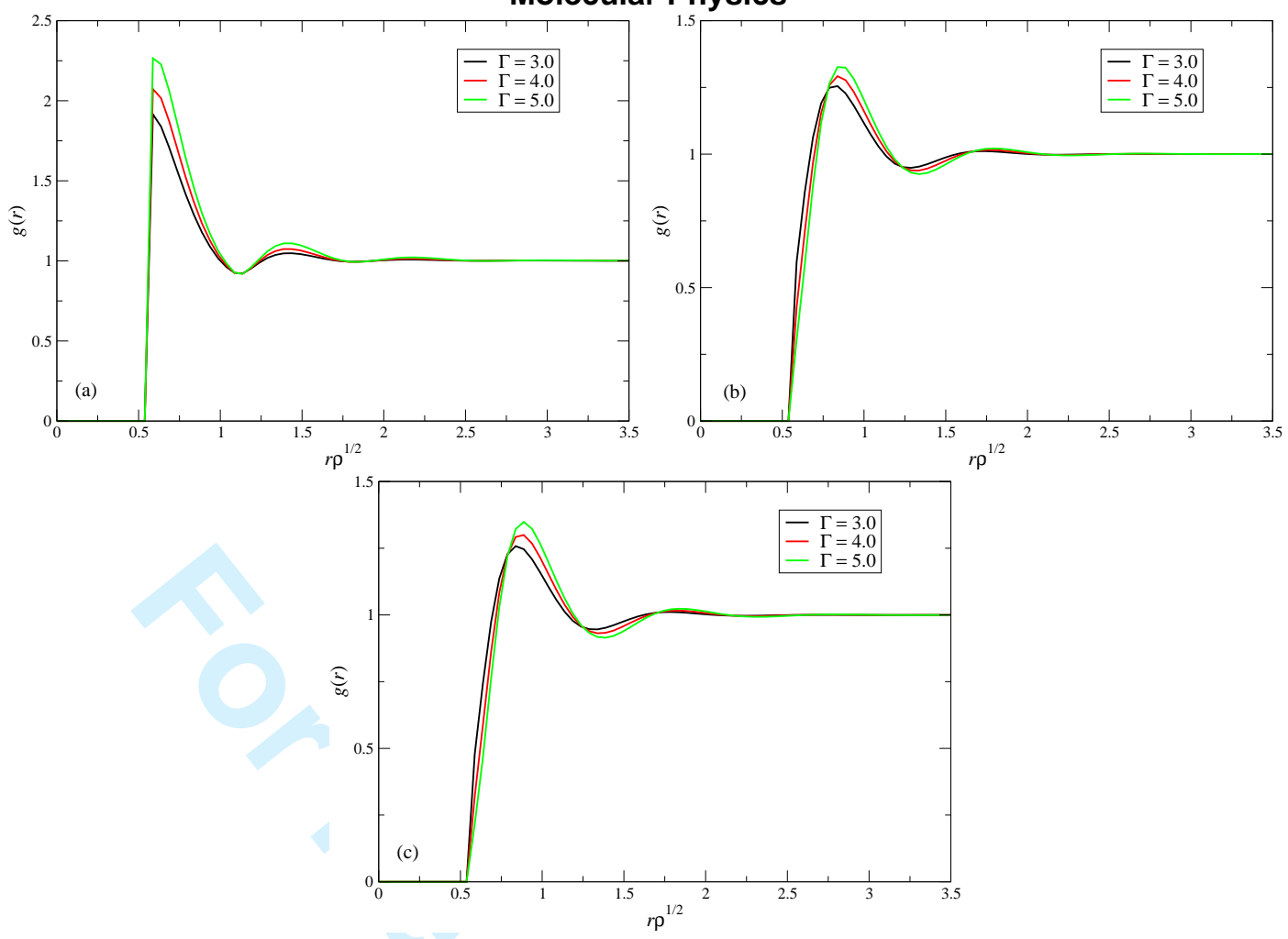

FIG. 2: The radial distribution function for different tilt and in-plane angles (a) $\phi=60^{\circ}, \theta_{\mathrm{R}}=0^{\circ}$, (b) $\phi=75^{\circ}, \theta_{\mathrm{R}}=30^{\circ}$, and (c) $\phi=85^{\circ}, \theta_{\mathrm{R}}=60^{\circ}$.

nearly unchanged. This behavior is physically plausible: the interaction strength can be increased by turning the magnetic field $\mathbf{B}_{0}$ stronger while the number of particles remains unchanged. Therefore, the typical inter-particle distance is not altered, but the degree of local ordering is affected, leading to a more pronounced liquid structure. Interesting is the peak position, which coincides with the hard-disk diameter for large tilt angles and lined-up configurations, fig. 2(a). The repulsion is too weak for a tilt angle $\phi=60^{\circ}$ and a head-to-tail configuration, so the particles feel the hard disc constraint.

Secondly, we can examine the local ordering of the particles for different in-plane directions while keeping the interaction strength and the tilt angle fixed. The results are shown in fig. 3 for different fixed tilt angles. In contrast to the case of a perpendicular field, the radial distribution functions for different in-plane directions do not coincide anymore. This effect is clearly traced back to the anisotropy of the interaction induced by the tilted magnetic field. We realize for all tilt angles that the local structure is most pronounced in direction of the in-plane component of the external field. The dipole-dipole interaction favors fully 

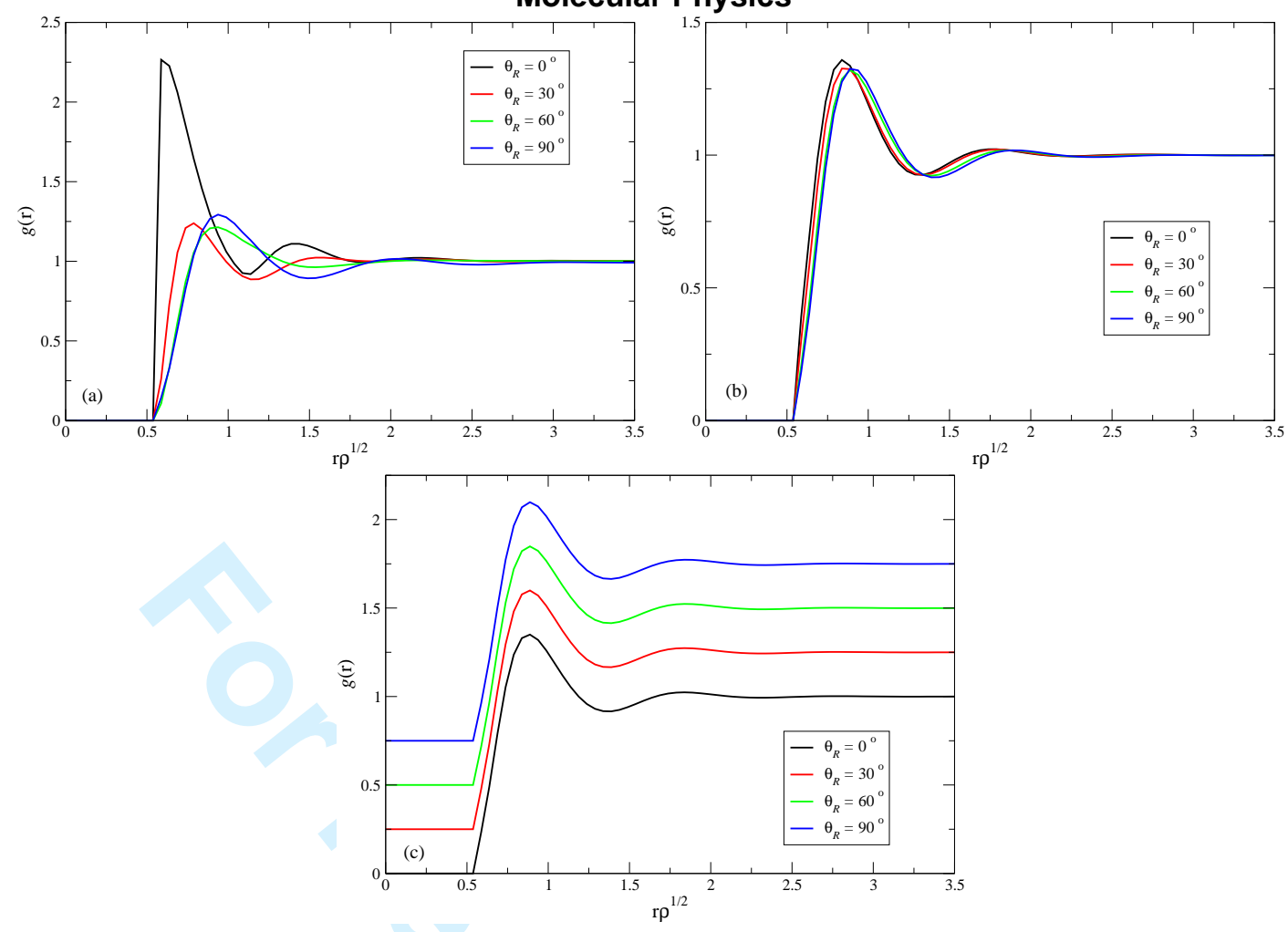

FIG. 3: The radial distribution function for fixed $\Gamma=5.0$ and different tilt angles $(\mathrm{a}) \phi=60^{\circ},(\mathrm{b})$ $\phi=75^{\circ}$, and (c) $\phi=85^{\circ}$. The curves of the latter plot have been shifted by 0.25 , respectively, for better distinction.

aligned moment, i.e., head-to-tail configuration, in agreement with previous results [19] and with the previously discussed ones. Furthermore, the deviation among the different local radial distribution functions increases with increasing tilt angle. The differences are most obviously observed for $\phi=60^{\circ}$ whereas for $\phi=85^{\circ}$ we barely recognize any significant distinctions. This can be understood by the growth of anisotropy with larger tilt angles.

Finally, we explicitly compare different tilt angles $\phi$ for fixed interaction strength and fixed in-plane angles $\theta_{\mathrm{R}}$. We present the results in fig. 4 for different fixed in-plane angles. Only for the in-plane direction of the magnetic field the structure is more pronounced the more the field is tilted. While always working in the repulsive regime, the repulsion between headto-tail configurations become weaker, leading to a stronger alignment among the particles in the field in-plane direction. This does not hold true for any other in-plane direction and therefore no predictions of the heights of the different local ordering can be made. It can be stated, though, that the anisotropy is rather weak in the $\phi$-regime between $75^{\circ}$ and $90^{\circ}$ but 

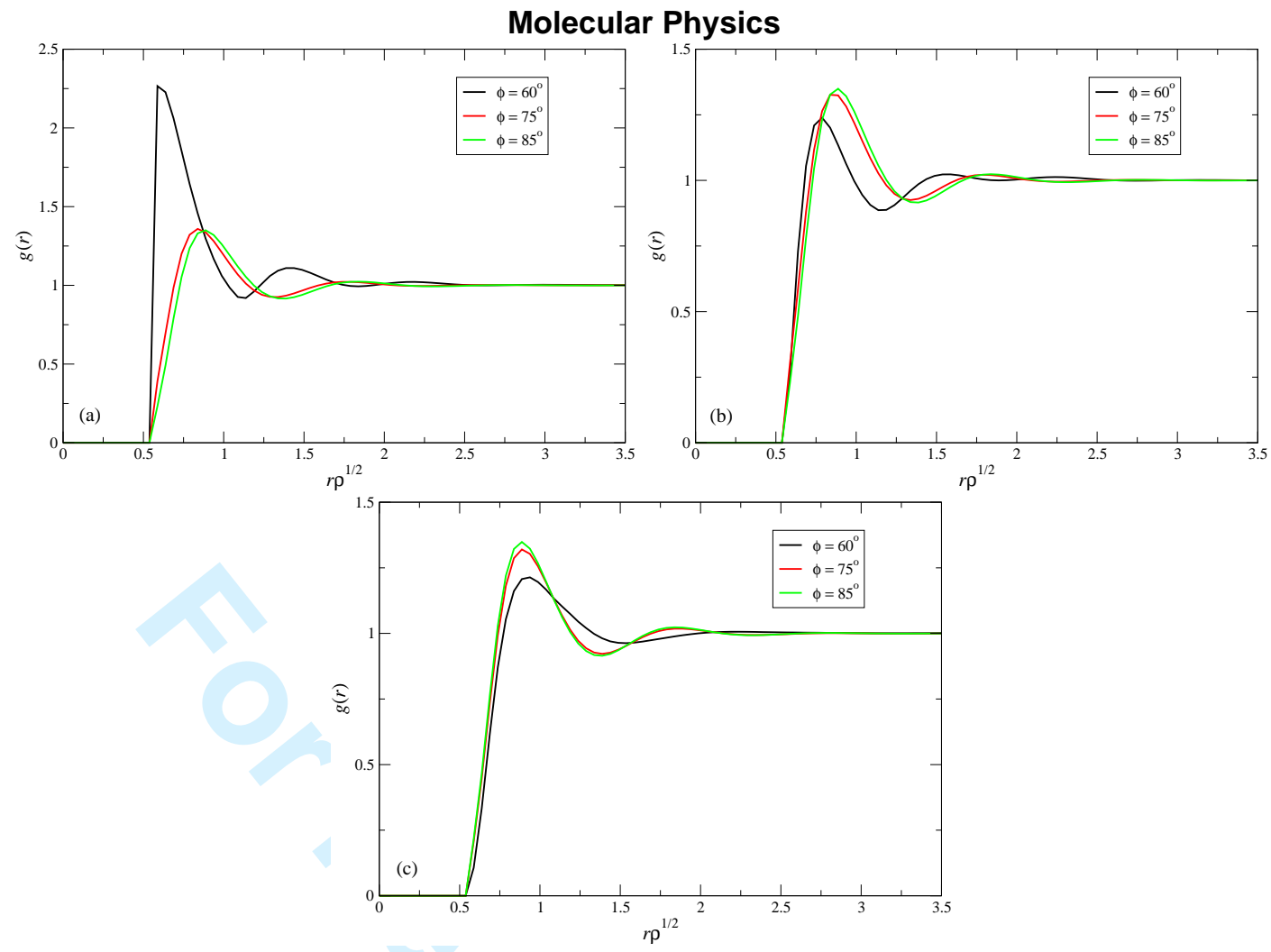

FIG. 4: The radial distribution function for fixed $\Gamma=5.0$ and different in-plane angles (a) $\theta_{\mathrm{R}}=0^{\circ}$, (b) $\theta_{\mathrm{R}}=30^{\circ}$, and (c) $\theta_{\mathrm{R}}=60^{\circ}$.

well pronounced for $\phi=60^{\circ}$.

\section{COMPARISON TO THE SOLID PHASE}

In this section, we compare the correlations in the above described fluid phase to that of the solid state which is stable at lower temperature at the same fixed tilt angle. Properties of the solid phase have been examined elsewhere $[17,20]$. In the ranges of tilt angles examined here a two-dimensional body-centered rectangular lattice was found to be stable. One can define two lattice constants, $a_{1}$ and $a_{2}$, where $a_{1}$ is parallel to the projected external field and $a_{2}$ points to the body-centered particle. For a perpendicular field the lattice constants are equal, $a_{1}=a_{2}$, but tilting the field yields an anisotropy, $a_{1} \neq a_{2}$. In general, $a_{1}<a_{2}$, which clearly stems from the fact that there is less repulsion in the direction parallel to the magnetic field.

First, we want investigate whether this anisotropy of the solid is anticipated by the 


\begin{tabular}{|c|c|c|}
\hline tilt angle $\phi$ & solid phase, $\Gamma=\infty$ & fluid phase, $\Gamma=5.0$ \\
\hline 90 & 1 & 1 \\
\hline 85 & 0.78 & 1.011 \\
\hline 75 & 0.8 & 1.006 \\
\hline 60 & $>1$ & 0.835 \\
\hline
\end{tabular}

TABLE I: Comparison of ratio of the "Lindemann ratios" in directions parallel and perpendicular to the magnetic field, both for the solid and fluid phases.

short-ranged order of the fluid. As equivalents of the lattice constants in the fluid state we consider the averaged peak position of the first maximum of $g(r)$ obtained by performing the first moment of $g(r)$ up to the first minimum. We examined this quantity for various $\theta_{R}$ directions. We have calculated these peak positions for $\theta_{R}=0$ and $\theta_{R}=60^{\circ}$ and have plotted their numbers in the inset of Figure 5. Their ratio is compared against the corresponding number of $a_{2} / a_{1}$ in the crystalline lattice at zero temperature [17]. We see the same kind of anisotropy. However, it is more pronounced in the solid than in the fluid state. This can be understood qualitatively since temperature is expected to smear out anisotropies.

The mean-square displacements of the particles in the directions parallel and perpendicular to the in-plane component of the external magnetic field have been probed by means of real-space experiments and harmonic lattice theory [20]. The anisotropy of the mean-square displacement behaves non-monotonically as a function of the tilt angle and does not correlate with the structural anisotropy of the crystal. We define a corresponding mean-square displacement in the fluid phase by calculating the second moments of $g(r)$ around the averaged peak positions. The roots of the different second moments are then divided by their corresponding peak position and considered as a "Lindemann" ratio in the fluid for various directions. The ratio of these "Lindemann" ratios for two directions parallel and perpendicular to the magnetic field is then given for various tilt angles in Table I. Comparing them to their solid counterparts from Ref. [20], we observe a different trend as a function of the tilt angle. The anisotropy of detailed structural quantities, therefore, is not the same in the fluid and solid phases. At the same time, it must be noted that whereas the Lindemann ratio has a well-defined meaning in the crystal phase, its definition in the fluid is somewhat arbitrary. 


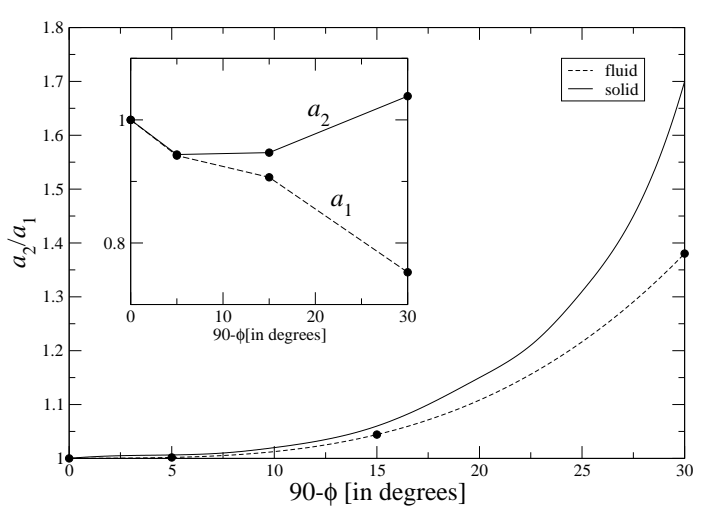

FIG. 5: The ratio of the lattice constants in direction of the lattice vectors as a function of the tilt angle $\phi$ of the external magnetic field for the solid phase (solid line) and the corresponding ratio for the fluid (dashed line). The inset shows peak positions $a_{1}$ and $a_{2}$ normalized to those of a perpendicular external field in the fluid phase.

\section{CONCLUSIONS}

In this paper, we have derived the formalism of integral equation theory for an anisotropic interaction. We have expanded all correlation functions in a set of orthogonal functions to cast the integral equation in a numerically accessible form. The cumbersome task of a two-dimensional, fast Fourier transform has been tackled by the introduction of Hankel transforms of higher orders. These could be in turn reduced to easily calculable 0th-order Hankel transforms.

Due to the anisotropy of the interaction with tilted magnetic field, the local ordering of the particles becomes also anisotropic. A tendency for alignment of particles in direction of the in-plane component of the magnetic field is observed, which is a precursor of the chain formation taking place when attractions set in. This strong ordering cannot be predicted by integral equation theory, since the iteration breaks down. Whether this is due to the very large of $m$-terms needed in the plane-wave expansion or it is a deeper problem of integral equations, remains a problem for the future. A comparison with the crystal which is stable at lower temperature shows that the anisotropy is similar to that in the fluid phase, but details like mean-square displacements possess opposite trends as a function of the tilt angle. 
Our study was limited to tilt angles which do not exceed the limit above which dipolar attractions between the particles show up. The reason lies in the fact that the HNC iteslf becomes unstable for such strong titl angles and fails to achieve converegnce. The degree of anisotropy for the angles we considered is still clearlz visible and correlated to ensuing crystal structures. Large tilt angles lead to the formation of columns of variable thickness, whose existence has been predicted by simulation [28] and confirmed experimentally [29]. A theoretical approach to explain the formation of these columns at high tilt angles is still missing and will be the subject of future work.

\section{ACKNOWLEDGMENTS}

The authors wish to thank Dominique Levesque, Ronald Blaak, and Martin Konieczny for helpful discussions. This work has been supported by the Deutsche Forschungsgemeinschaft within the Collaborative Research Center SFB-TR6, "Physics of Colloidal Dispersions in External Fields", (Project Section C3), and by the European Centre of Excellence "Softcomp".

\section{APPENDIX}

In this appendix, we show a strict derivation of the interconnection between the coefficients of the series expansion in real and reciprocal space. Performing the Fourier transform of the direct correlation function $c(r)$ leads to the following expressions:

$$
\begin{aligned}
\tilde{c}(\mathbf{k}) & =\int \mathrm{d}^{2} r c(\mathbf{r}) \exp (\mathrm{ik} \cdot \mathbf{r}) \\
& =\int \mathrm{d}^{2} r \sum_{m} c_{m}(r) \exp \left(-\mathrm{i} m \theta_{R}\right) \exp \left[\mathrm{i} k r \cos \left(\theta_{R}-\theta_{K}\right)\right] \\
& =\sum_{m} \int_{0}^{\infty} \mathrm{d} r r c_{m}(r) \int_{0}^{2 \pi} \mathrm{d} \theta_{R} \exp \left[-\mathrm{i} m\left(\theta_{R}-\theta_{K}\right)\right] \exp \left[\mathrm{i} k r \cos \left(\theta_{R}-\theta_{K}\right)\right] \exp \left(-\mathrm{i} m \theta_{K}\right) \\
& =\sum_{m} \exp \left(-\mathrm{i} m \theta_{K}\right) \int_{0}^{\infty} \mathrm{d} r r c_{m}(r) \underbrace{\int_{0}^{2 \pi} \mathrm{d} \theta_{R} \exp \left[-\mathrm{i} m\left(\theta_{R}-\theta_{K}\right)\right] \exp \left[\mathrm{i} k r \cos \left(\theta_{R}-\theta_{K}\right)\right]}_{I(k r)} .
\end{aligned}
$$

We put $x \equiv \theta_{R}-\theta_{K}$, and substitute

$$
I(k r)=\int_{-\theta_{K}}^{2 \pi-\theta_{K}} \mathrm{~d} x \exp (-\mathrm{i} m x) \exp (\mathrm{i} k r \cos x),
$$


using the $2 \pi$-periodicity of the integrand, we write

$$
I(k r)=\int_{0}^{2 \pi} \mathrm{d} x \exp (-\mathrm{i} m x) \exp (\mathrm{i} k r \cos x) \equiv 2 \pi \mathrm{i}^{m} J_{m}(k r) .
$$

Then, we can write the Fourier transform as:

$$
\begin{aligned}
\tilde{c}(k) & =\sum_{m} \exp \left(-\mathrm{i} m \theta_{K}\right) 2 \pi \mathrm{i}^{m} \int_{0}^{\infty} \mathrm{d} r r c_{m}(r) J_{m}(k r) \\
& =\sum_{m} \tilde{c}_{m}(k) \exp \left(-\mathrm{i} m \theta_{K}\right)
\end{aligned}
$$

with

$$
\tilde{c}_{m}(k)=2 \pi \mathrm{i}^{m} \int_{0}^{\infty} \mathrm{d} r r c_{m}(r) J_{m}(k r) .
$$

Similarly, we obtain

$$
c_{m}(r)=(2 \pi)^{-1} \dot{\mathrm{i}}^{m} \int_{0}^{\infty} \mathrm{d} k k \tilde{c}_{m}(k) J_{m}(k r) .
$$

The coefficients in real and reciprocal space can be transformed into each other by a simple Hankel transform of $m$ th-order.

[1] HANSEN, J.-P., and MCDONALD, I. R., 2006, Theory of Simple Liquids, 3rd ed., (London:Academic).

[2] GRAY, C. G., and GUBBINS, K. E., 1984, Theory of Molecular Fluids (Oxford: Clarendon Press).

[3] CAnessa, E., DAguanno, B., WeYerich, B., and KLEIn, R., 1991, Mol. Phys., 73, 175.

[4] BRESME, F., 1998, J. Chem. Phys., 108, 4505.

[5] KLAPP, S. H. L., 2005, J. Phys.: Condens. Matter, 17, R525.

[6] KLAPP, S., and FORSTMAnN, F., 1999, Phys. Rev. E, 60, 3183.

[7] LADO, F., LOMBA, E., and MARTIN, C., 2004, J. Mol. Liq., 112, 51.

[8] ZAHN, K., LENKE, R., and MARET, G., 1999, Phys. Rev. Lett., 82, 2721.

[9] ZAHN, K., and MARET, G., 2000, Phys. Rev. Lett., 85, 3656.

[10] WILle, A., VAlmont, F., ZAHN, K., and MARET, G., 2002, Europhys. Lett., 57, 219.

[11] ZAHN, K., WILle, A., MARET, G., SENGUPTA, S., and NIELABA, P., 2003, Phys. Rev. Lett., 90, 155506. 
[12] ZAHN, K., and MARET, G., 1999, Curr. Opin. Colloid Interface Sci., 4, 60.

[13] TAVARES, J. M., WEIS, J. J., and TELO DA GAMA, M. M., 2006, Phys. Rev. E, 73, 041507.

[14] RINN, B., ZAHN, K., MAASS, P., and MARET, G., 1999, Europhys. Lett., 46, 537.

[15] HOFFMAnN, N., EBERT, F., LIKOS, C. N., LÖWEN H., and MARET, G., 2006, Phys. Rev. Lett., 97, 078301.

[16] HOFFMAnn, N., LIKOS, C. N., and LÖWEN, H., 2006, J. Phys.: Condens. Matter, 18, 10193.

[17] EISEnMAnN, C., KEIM, P., GASSER, U., et al., 2004, J. Phys.: Condens. Matter, 16, S4095.

[18] EISEnMann, C., GASSER, U., KEIM, P., and MARET, G., 2004, Phys. Rev. Lett., 93, 105702.

[19] Froltsov, V. A., BlAAK, R., LIKOS, C. N., and LÖWEN, H., 2003, Phys. Rev. E, 68, 061406.

[20] Froltsov, V.A., LIKOS, C.N., LÖWEn, H., EISENMANn, C., GASSER, U., KEIM, P., and MARET, G., 2005, Phys. Rev. E, 71, 031404.

[21] CAIllol, J. M., WEIS, J. J., and PATEY, J. M., 1988, Phys. Rev. A, 38, 4772.

[22] CAILlOL, J. M., LEVESQUE, D., and WEIS, J. J., 1981, Mol. Phys., 44, 733.

[23] FRIES, P.H., and PATEY, G.N., 1985, J. Chem .Phys., 82, 429.

[24] LADO, F., 1982, Mol. Phys., 2, 283.

[25] LADO F., J. Comput. Phys., 8, 417.

[26] ABRAMOWITZ, M., and STEGUN, I.A. (eds), 1972, Handbook of Mathematical functions (New York: Dover).

[27] LI, J., J. Comput. Anal. Appl., 183, 29.

[28] WEIS, J. J., 2005, Mol. Phys., 103, 7

[29] KLOKKEnBURG, M., ERNE, B. H., MEELDIJK, J. D., WIEDENMANN, A., Petukhov, A. V., Dullens, R. P. A., and PHIlPiSe, A. P., 2006, Phys. Rev. Lett., 97, 185702 . 
B

$\mathbf{B}_{\perp}$
1
2
3
4
5
6
7
8
9
10
11
12
13
14
15
16
17
18
19
20
21
22
23
24
25
26
27
28
29
30
$3(\mathbf{a}$
3
a

$\mathrm{B}_{\|}$

a)
Molecular Physics

Page 22 of 33

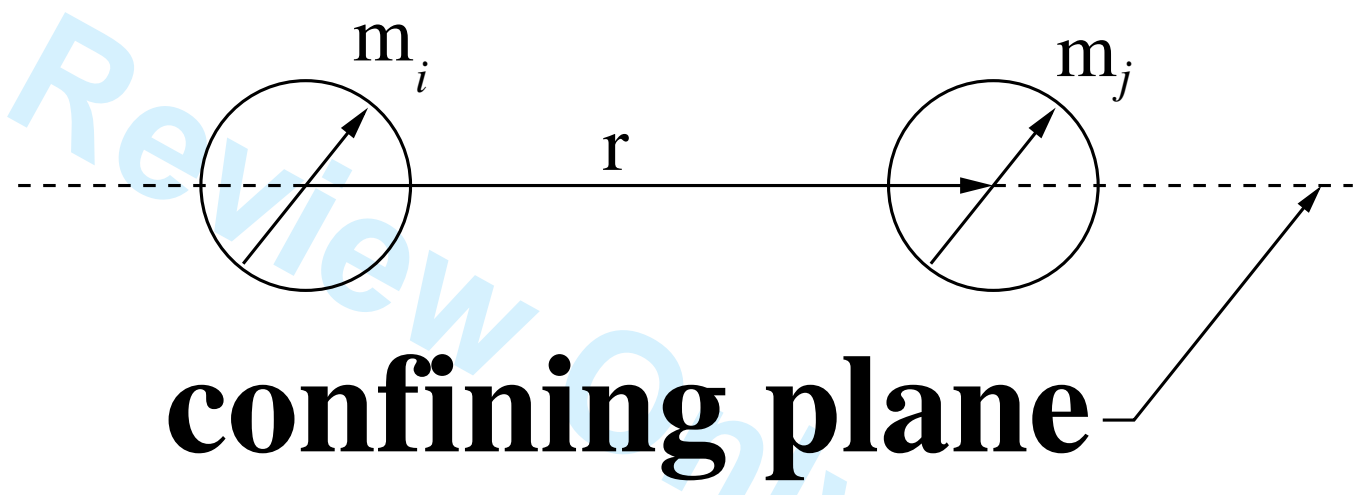

URL: http://mc.manuscriptcentral.com/tandf/tmph 


\section{Page 23 of 33}

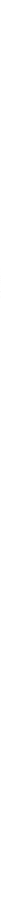




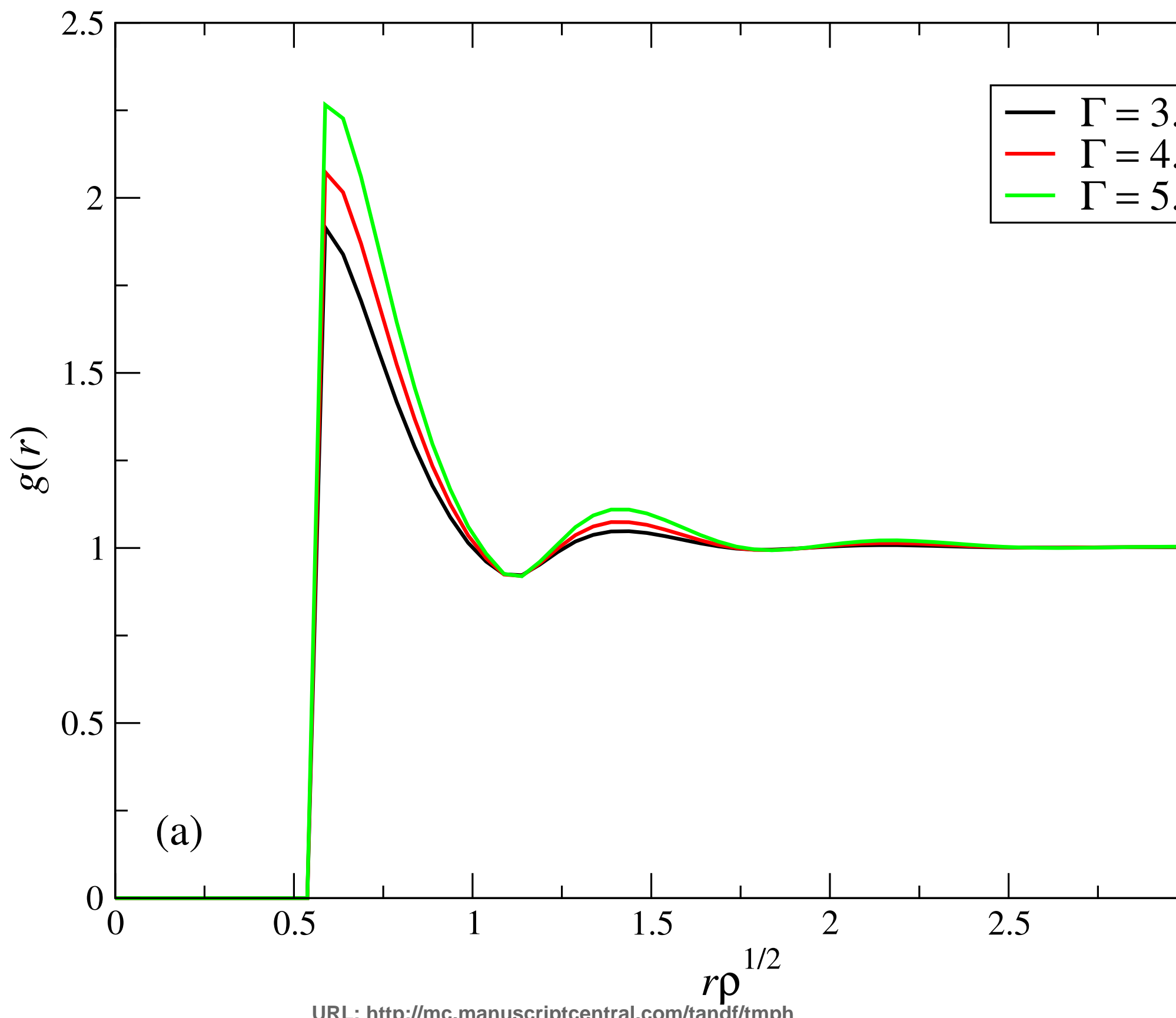

URL: http://mc.manuscriptcentral.com/tandf/tmph 


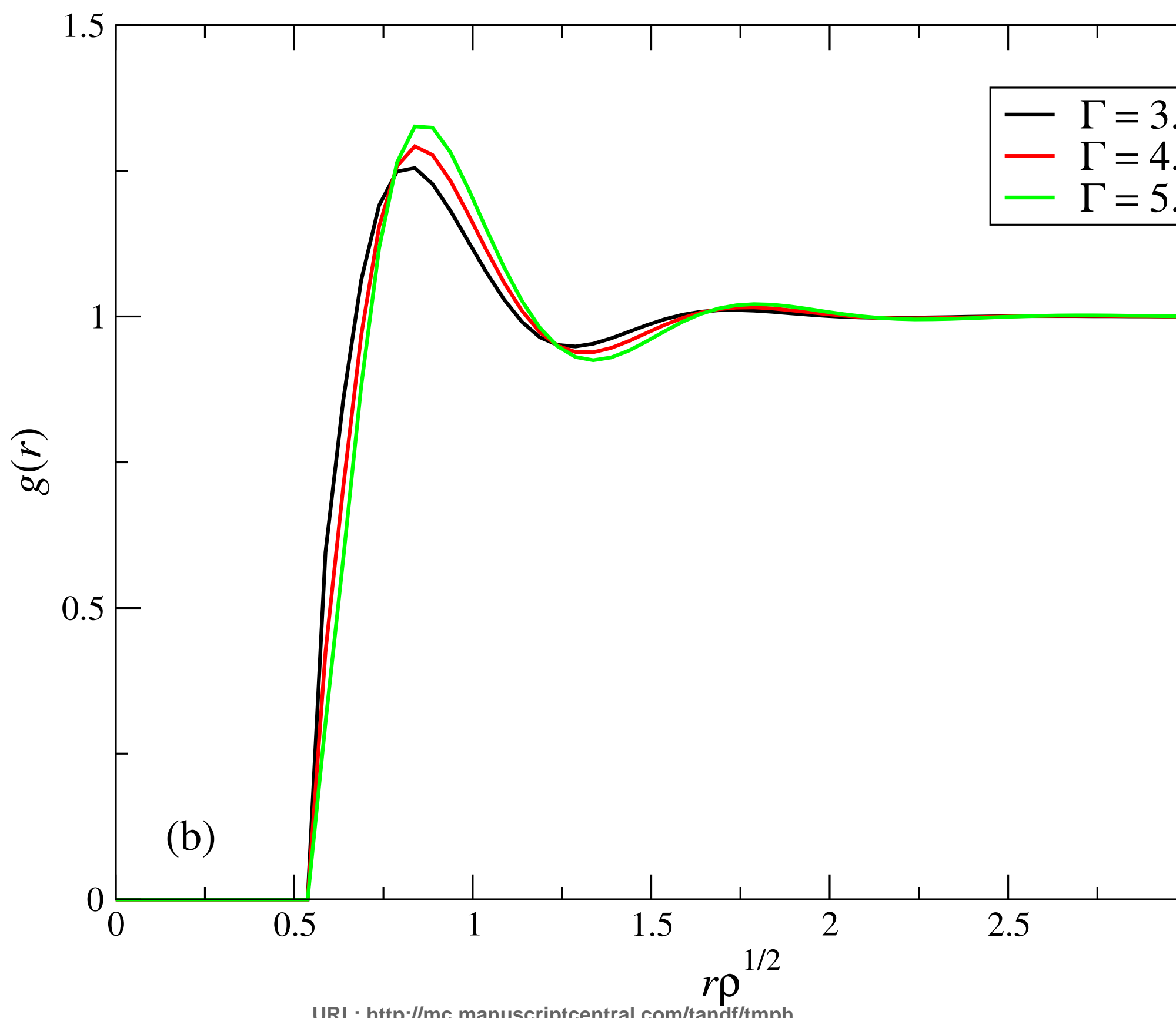

URL: http://mc.manuscriptcentral.com/tandf/tmph 


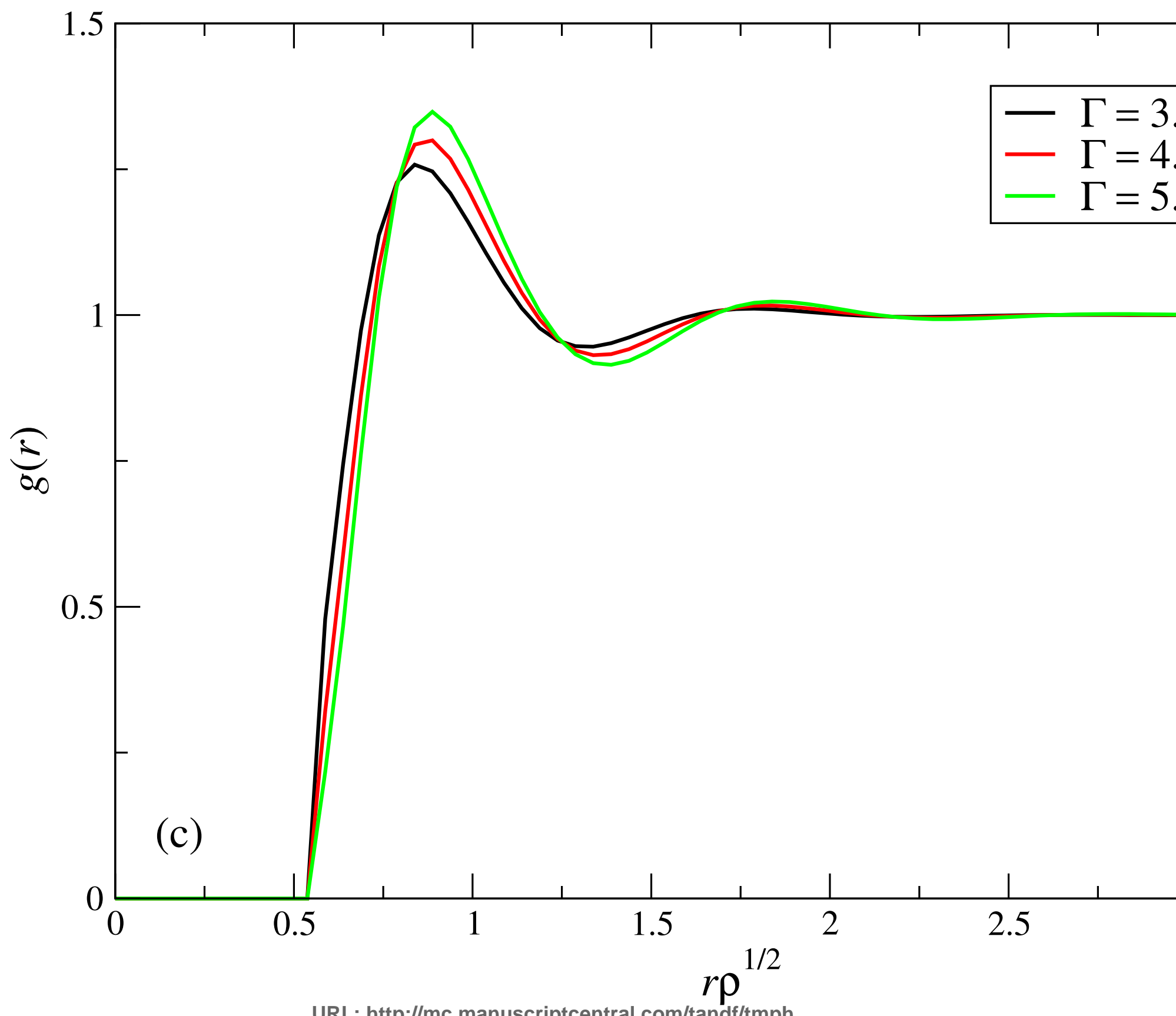

URL: http://mc.manuscriptcentral.com/tandf/tmph 


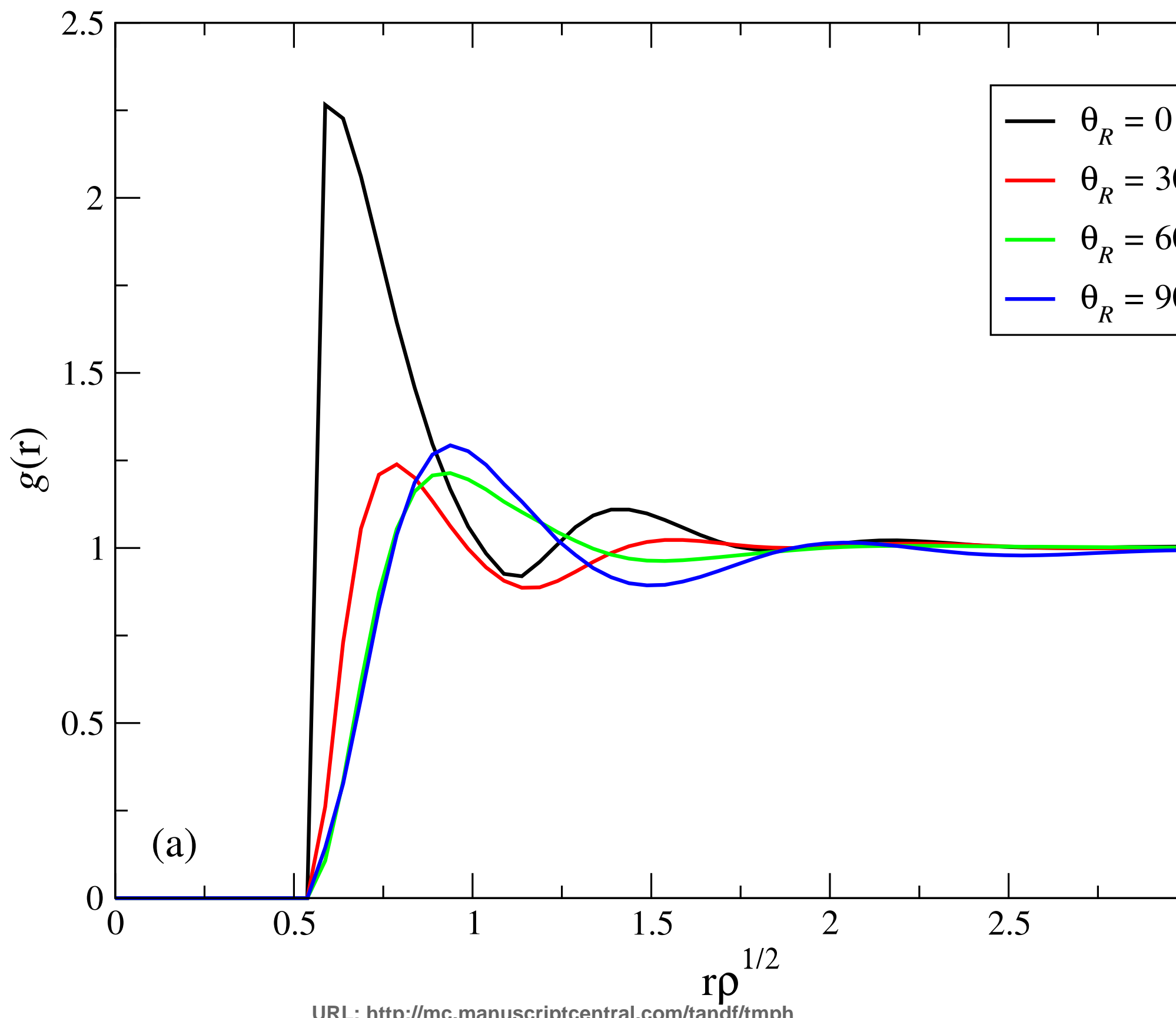

URL: http://mc.manuscriptcentral.com/tandf/tmph 


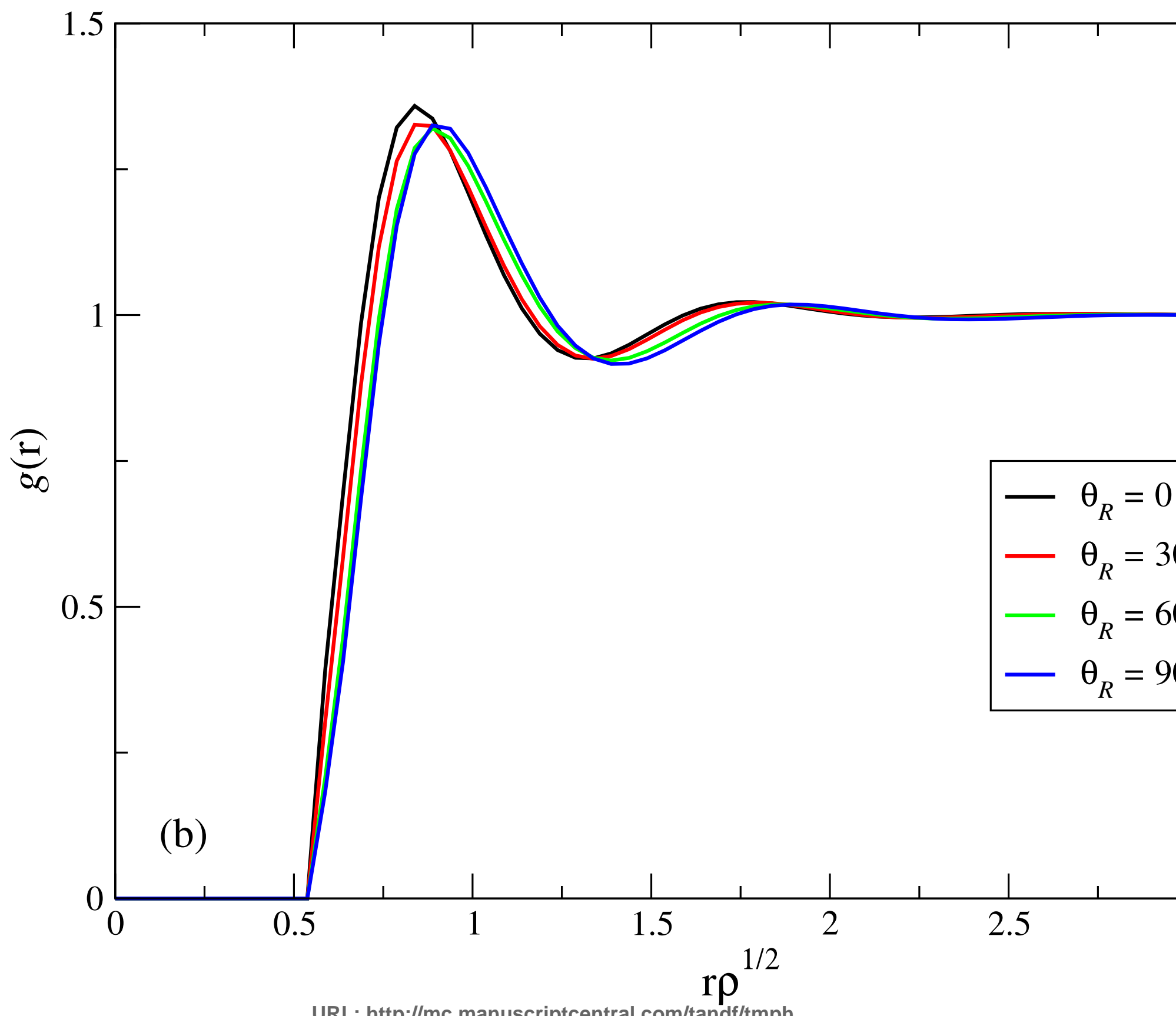

URL: http://mc.manuscriptcentral.com/tandf/tmph 


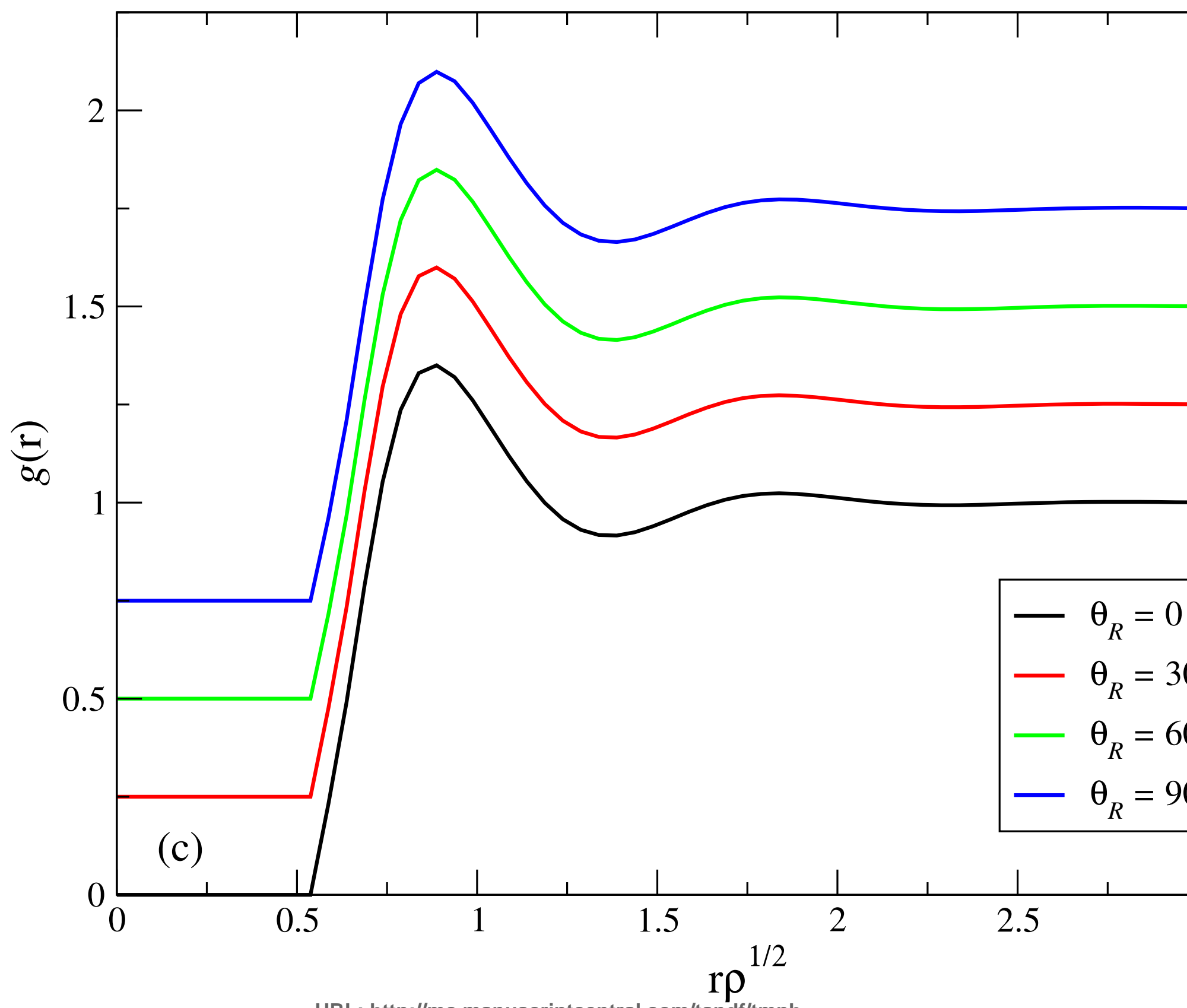

URL: http://mc.manuscriptcentral.com/tandf/tmph 


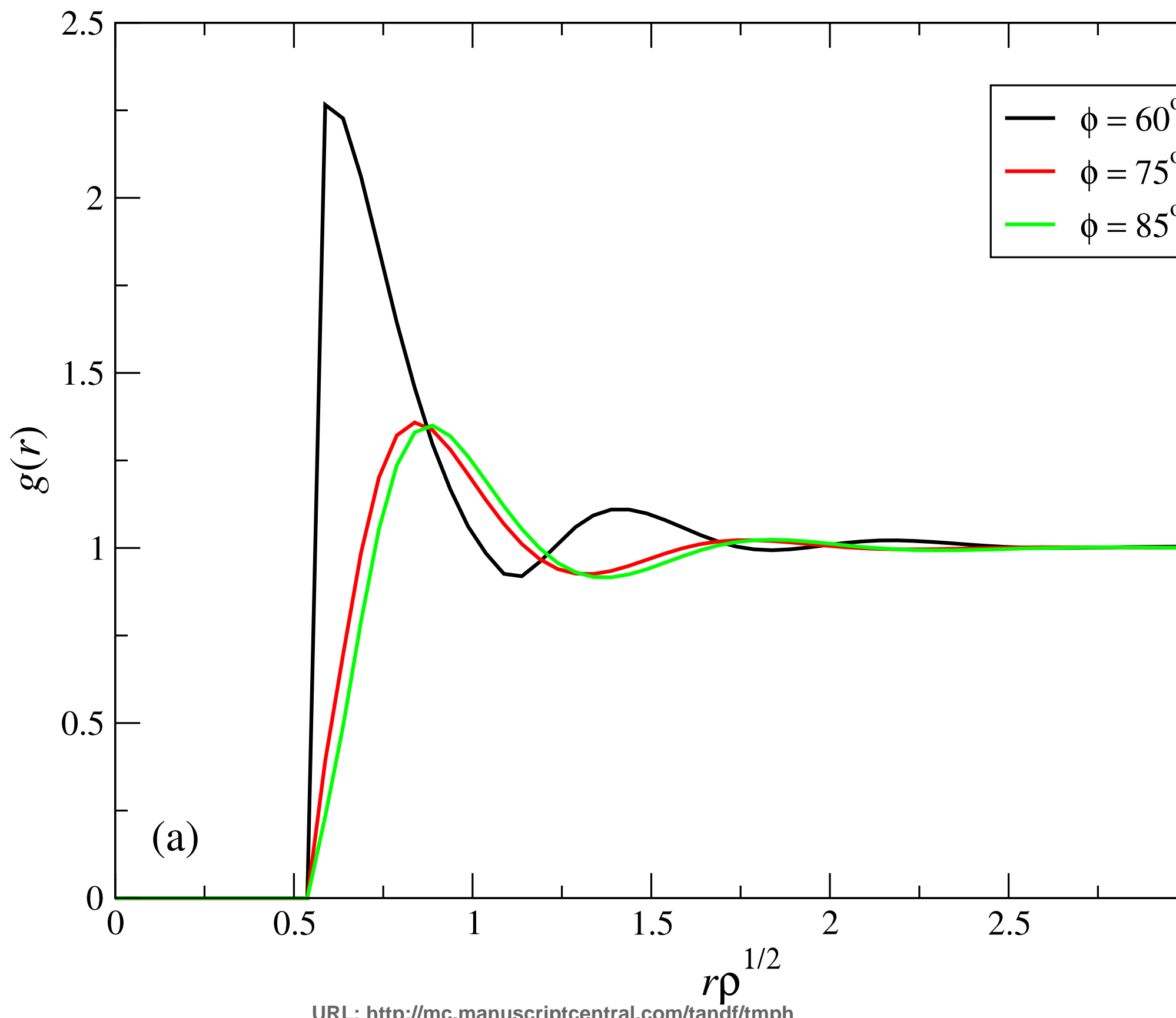

URL: http://mc.manuscriptcentral.com/tandf/tmph 


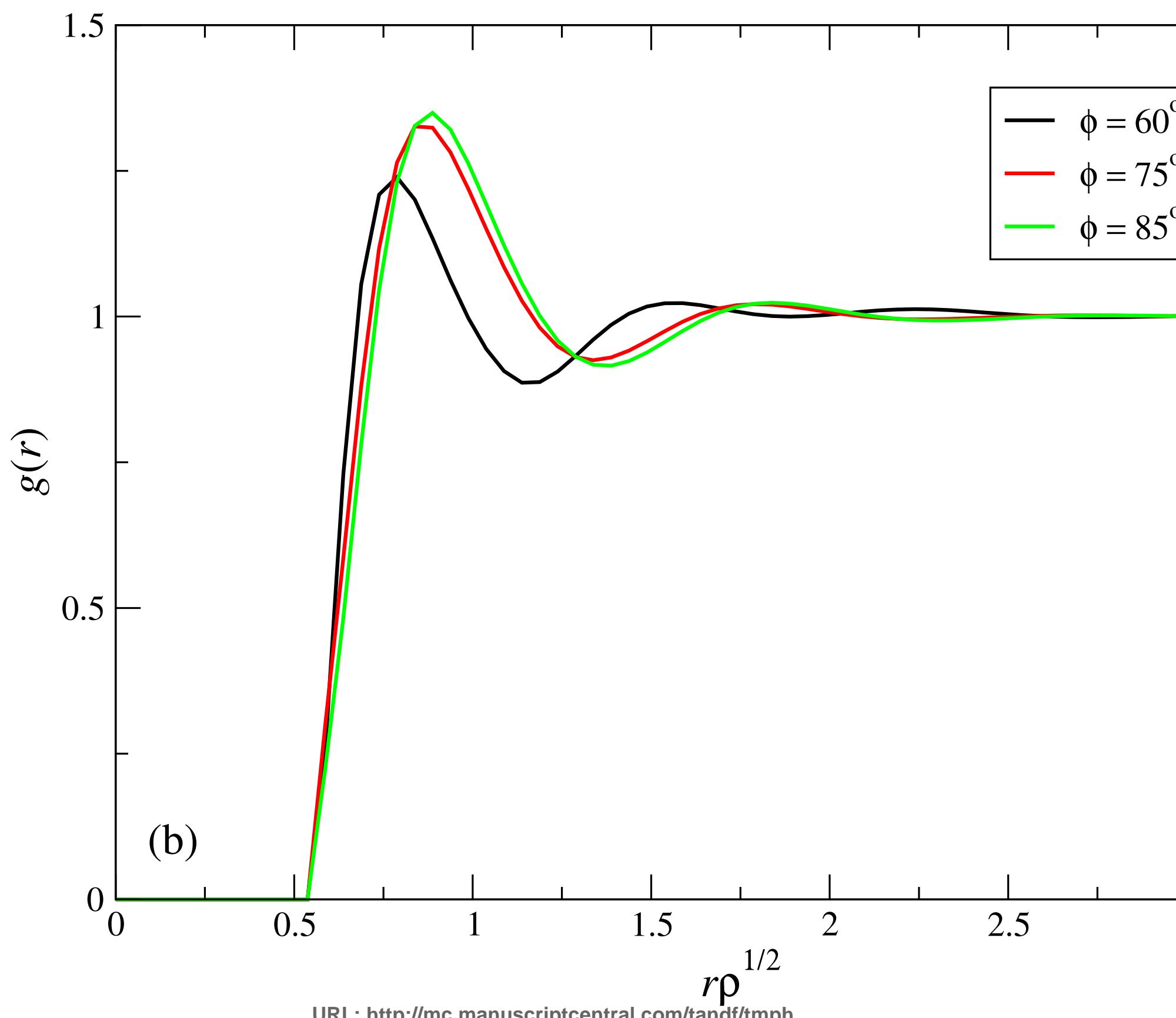

URL: http://mc.manuscriptcentral.com/tandf/tmph 


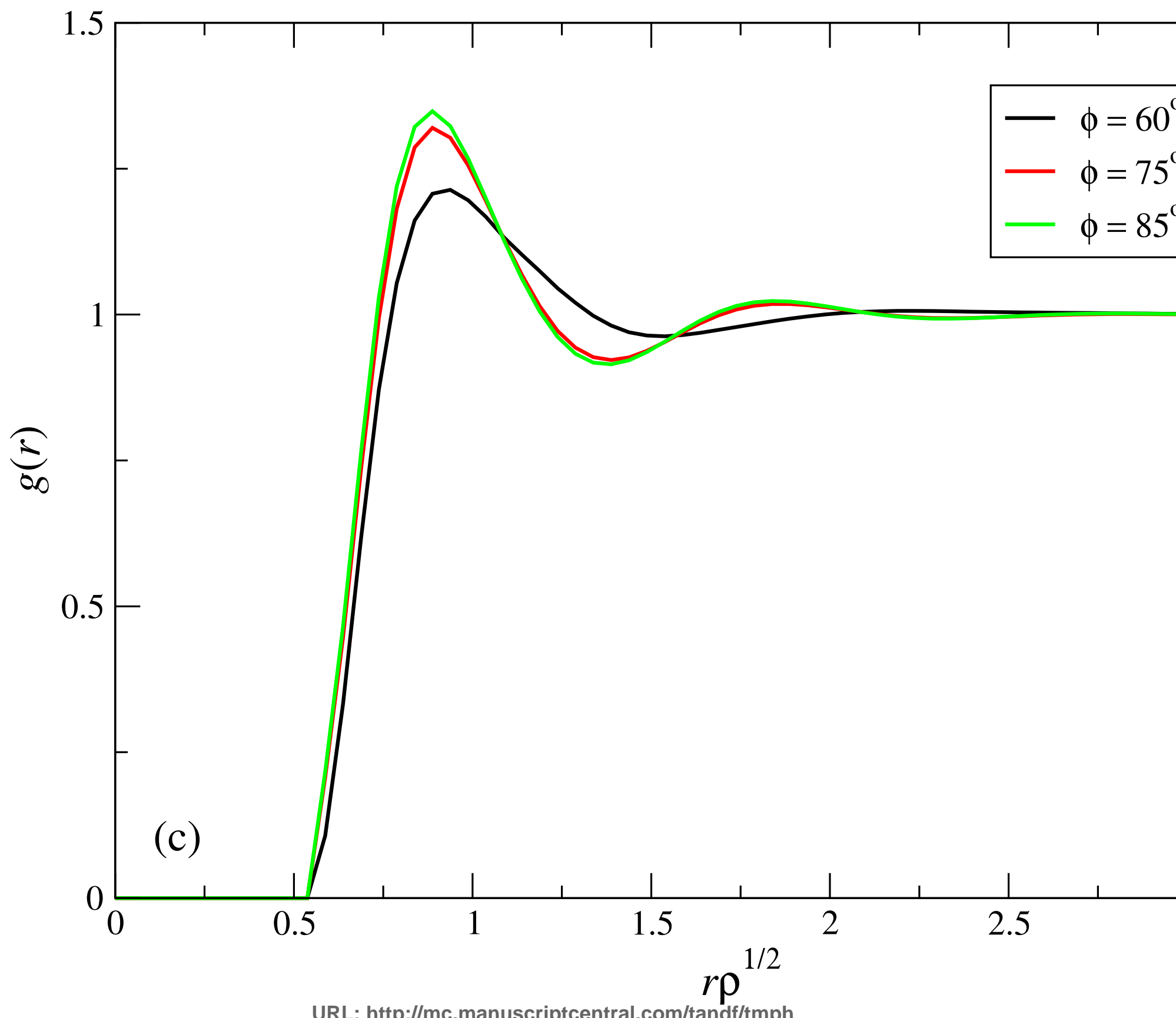

URL: http://mc.manuscriptcentral.com/tandf/tmph 


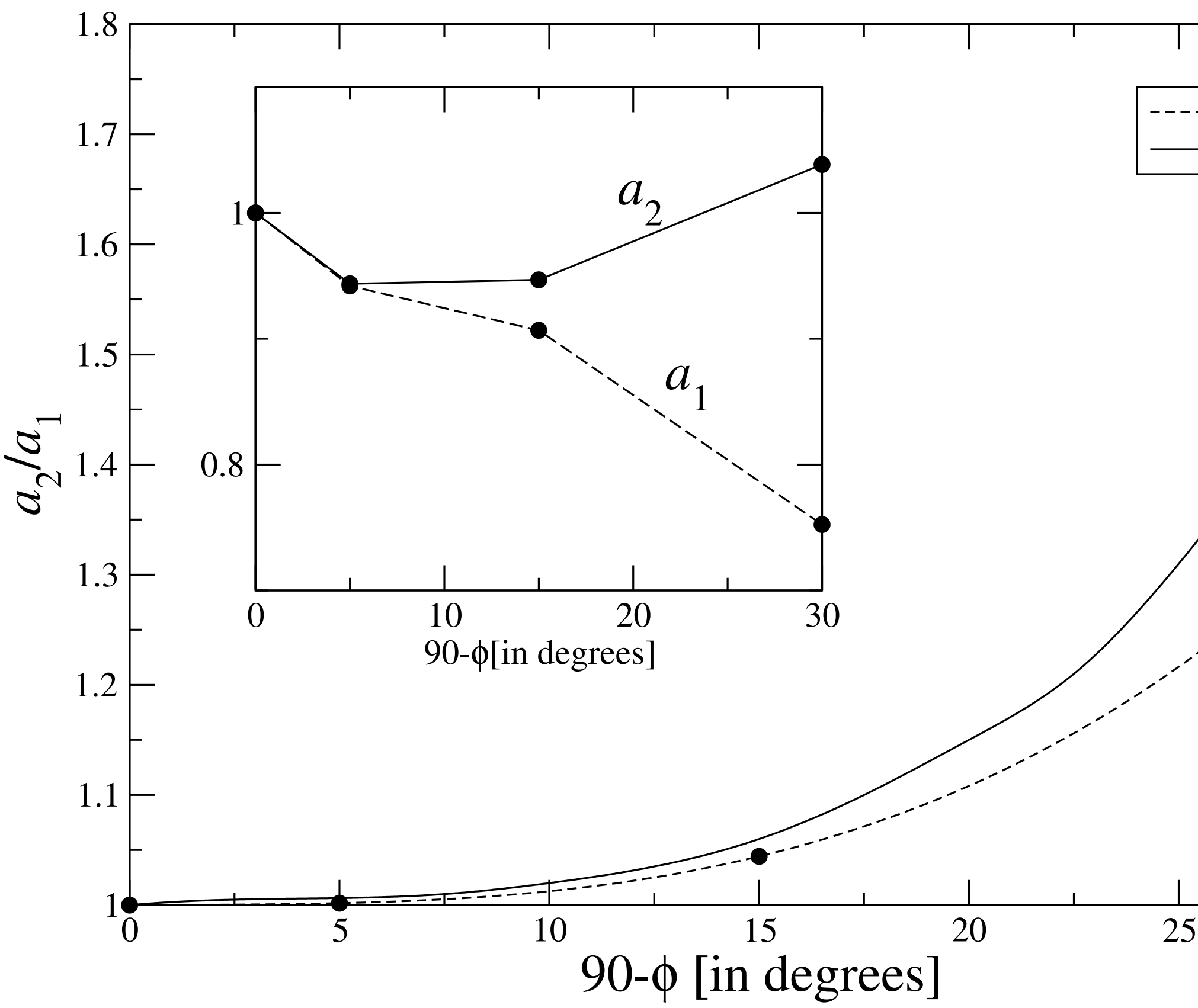

URL: http://mc.manuscriptcentral.com/tandf/tmph 